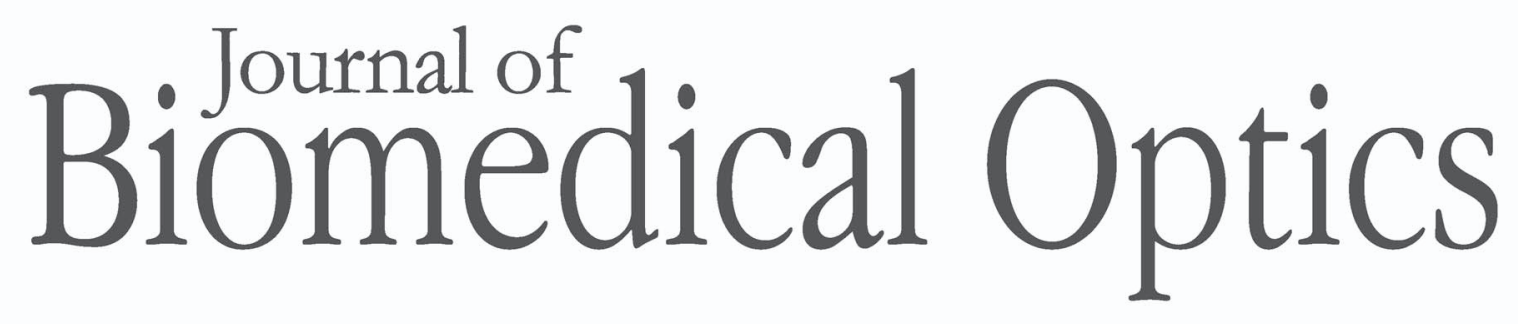

SPIEDigitalLibrary.org/jbo

\title{
Spatial sensitivity of acousto-optic and optical near-infrared spectroscopy sensing measurements
}

Sonny Gunadi

Terence S. Leung 


\title{
Spatial sensitivity of acousto-optic and optical near-infrared spectroscopy sensing measurements
}

\author{
Sonny Gunadi and Terence S. Leung \\ University College London, Department of Medical Physics and Bioengineering, Malet Place Engineering Building, \\ London, WC1E 6BT, United Kingdom
}

\begin{abstract}
Near-infrared spectroscopy (NIRS) is a popular sensing technique to measure tissue oxygenation noninvasively. However, the region of interest (ROI) is often beneath a superficial layer, which affects its accuracy. By applying focused ultrasound in the ROI, acousto-optic (AO) techniques can potentially minimize the effect of physiological changes in the superficial layer. Using absorption perturbation experiments in both transmission and reflection modes, we investigated the spatial sensitivity distributions and mean penetration depths of an AO system based on a digital correlator and two popular NIRS systems based on i. intensity measurements using a single source and detector configuration, and ii. spatially resolved spectroscopy. Our results show that for both transmission and reflection modes, the peak relative sensitivities of the two NIRS systems are near to the superficial regions, whereas those of the $\mathrm{AO}$ technique are near to the ROIs. In the reflection mode, when the ROI is deeper than $14 \mathrm{~mm}$, the AO technique has a higher absolute mean sensitivity than the two NIRS techniques. As the focused ultrasound is moved deeper into the turbid medium, the mean penetration depth increases accordingly. The focused ultrasound can shift the peak relative sensitivity of the AO measurement toward its focused region. (C) 2011 Society of Photo-Optical Instrumentation Engineers (SPIE). [DOI: 10.1117/1.3660315]
\end{abstract}

Keywords: acousto-optics; ultrasound modulated optics; near infrared spectroscopy; diffuse optics.

Paper 11369PRR received Jul. 13, 2011; revised manuscript received Oct. 21, 2011; accepted for publication Oct. 24, 2011; published online Nov. 23, 2011.

\section{Introduction}

Biological tissues have relatively low optical absorption in the near-infrared (NIR) range and therefore NIR light can penetrate deeper than the visible light into the tissue. Also in the NIR range, oxy- and deoxy-haemoglobin have distinctive absorption spectra, which allow their concentrations to be estimated using spectroscopy techniques. These two factors form the basis of a tissue oxygenation measurement technique generally known as NIR spectroscopy (NIRS), which has been widely used to perform noninvasive cerebral and muscle oxygenation measurements. ${ }^{1}$ The simplest form of NIRS involves only a single continuous-wave (cw) optical source and a detector placed several centimeters away in reflection mode. In this setup, NIR light travels $\sim 1$ to $2 \mathrm{~cm}$ deep into the tissue and is reflected back to the detector. The photon path distribution between the source and detector resembles a widely known "banana" shape. ${ }^{2}$ This detection scheme only allows the measurement of changes in the concentration of oxy- and deoxy-haemoglobin but not the absolute concentration or tissue oxygen saturation $\left(\mathrm{S}_{\mathrm{t}} \mathrm{O}_{2}\right)$. In recent years, this single source and detector (SSD) scheme became very popular in functional NIRS studies in which multiple channels of SSDs have been used to provide an activation map of the brain during certain functional tasks. An example of such a system is the ETG-4000 (Ref. 3) from Hitachi Medical Systems.

To provide an absolute $\mathrm{S}_{\mathrm{t}} \mathrm{O}_{2}$ measurement, spatially resolved (SR) spectroscopy has been used. This technique measures

Address all correspondence to: Terence Leung, University College London, Department of Medical Physics and Bioengineering, Malet Place Engineering Building, Gower Street, London, WC1E 6BT, United Kingdom. Tel: 44207679 0272; Fax: 44207679 0255; E-mail: ts|@medphys.ucl.ac.uk. reflected light in a number of detection points, which are used to calculate the attenuation slope. Using a solution of the diffusion equation, the attenuation slope can be converted into the absorption coefficient (scaled by a constant) which can then be used to calculate a $\mathrm{S}_{\mathrm{t}} \mathrm{O}_{2}$ measurement using a spectroscopy technique. ${ }^{4}$ Commercial NIRS monitors exploiting the SR approach include the NIRO series oxygenation monitors ${ }^{4}$ from Hamamatsu Photonics KK and the PortaMon NIRS system ${ }^{5}$ from Artinis Medical Systems.

The SSD and SR techniques are not without limitations. The oxygenation measurement site often has a layered structure and the region of interest (ROI) is beneath a superficial layer. In the case of functional NIRS or cerebral $\mathrm{S}_{\mathrm{t}} \mathrm{O}_{2}$ measurement, for instance, the ROI is the brain and yet there are layers of scalp, skull, and cerebrospinal fluid between the optical devices and the brain. This superficial layer has great influence on the oxygenation measurement. For the SSD case, computer modeling using Monte Carlo simulations and finite element method on an adult human head model have shown that the optical measurement is most sensitive to changes in the superficial layer instead of those in the ROI. ${ }^{6-8}$ This is in fact quite easy to understand: intuitively, an optical measurement is naturally more sensitive to a change near the source or the detector. The implication is that an intended NIRS measurement of the ROI is only valid when there is no significant physiological change (e.g., oxygenation and blood flow/volume changes) in the superficial layer.

One potential approach to increase the sensitivity in the ROI is to incorporate focused ultrasound with NIR light. This technique is known as acousto-optics (AO) or ultrasound modulated optical tomography and has been proposed as an imaging

$1083-3668 / 2011 / 16(12) / 127005 / 10 / \$ 25.00$ @ 2011 SPIE 


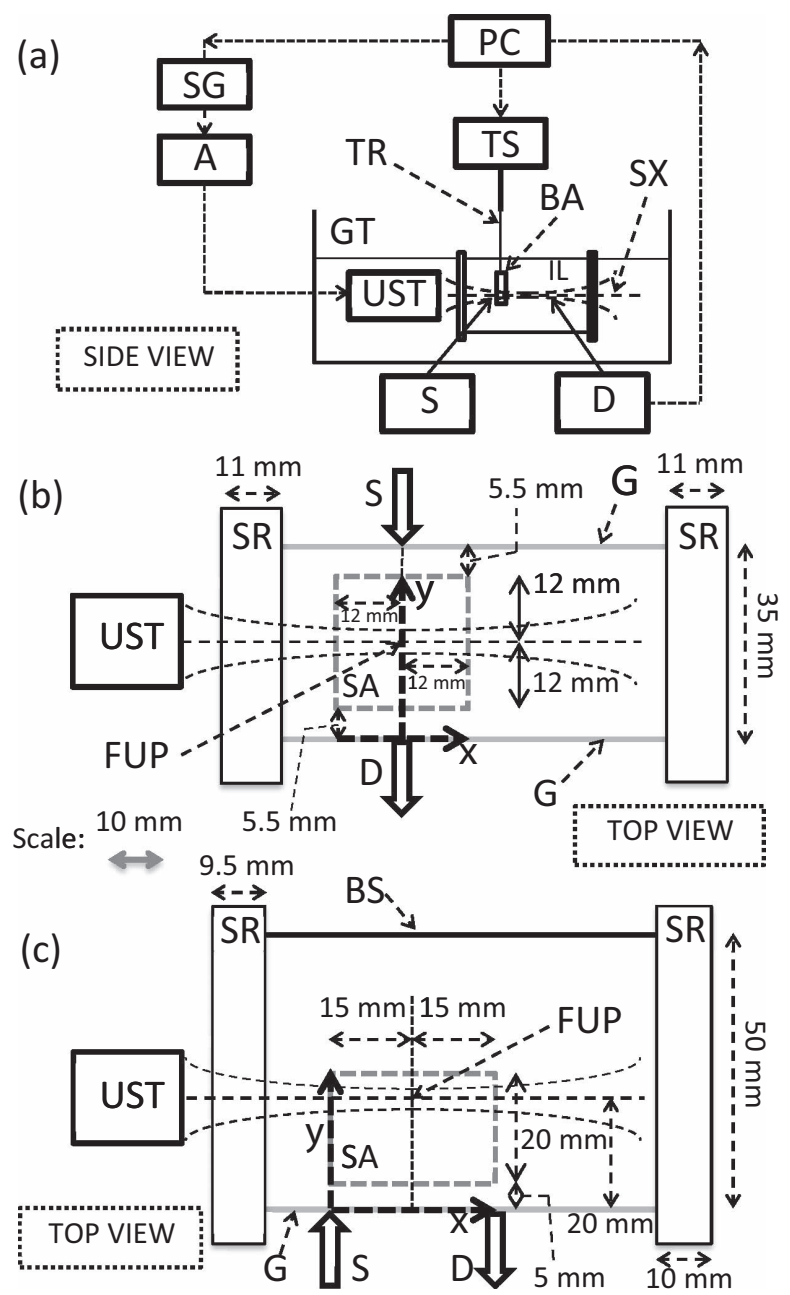

Fig. 1 (a) Key components of the experimental setup (side view); (b) transmission mode: distance between $\mathrm{S}$ and $\mathrm{D}$ is $35 \mathrm{~mm}$; (c) reflection mode: distance between $\mathrm{S}$ and $\mathrm{D}$ is $30 \mathrm{~mm}$ (top view). [UST: ultrasound transducer, S: optical source, D: optical detector, IL: intralipid solution, BA: black absorber, SX: scanning axis, GT: clear glass tank, TS: motorized translation stages, TR: thin metallic rod, A: RF amplifier, SG: signal generator, PC: computer, FUP: focused ultrasound peak, SR: silicon rubber, SA: scanning area, G: clear glass (3-mm thick), BS: black surface, $x$ : $x$-axis, $y$ : $y$-axis.]

technique for biological tissues. ${ }^{9}$ The principle is that focused ultrasound (US) alters the path length and refractive index locally in the US focus (ROI). ${ }^{9}$ When coherent NIR light travels through this ROI, the phase of the light will be systematically modulated. Some liken this process to the light being "tagged." On the surface of the measurement site, an optical speckle pattern is formed by the interferences between US modulated and unmodulated light. The speckle pattern is time-varying at the frequency of the focused US and its magnitude is indicative of the optical absorption and scattering coefficients in the US focus. By scanning the US focus, an image can be formed which shows the optical and acoustic properties of the turbid medium. ${ }^{10-13}$ Recently, Bratchenia et al. ${ }^{14}$ have combined AO with diffuse optical tomography to reconstruct images containing the optical properties of a turbid medium. Although originally proposed as an imaging technique, $\mathrm{AO}$ has great potential as a sensing tech- nique because of its capability to "tag" light and hence increases the sensitivity of the AO measurement in the ROI.

In order to assess the spatial sensitivity of an optical detection scheme, a setup involving a local absorber in an otherwise homogeneous turbid medium is often used..$^{6,15-17}$ The idea is that the local absorber (with a high absorption coefficient) in a particular location absorbs all the photons passing through it. As a result, the reduction in the detected intensity is proportional to the local photon path distribution ${ }^{18}$ or the photon measurement density function ${ }^{6}$ which can also be considered as the sensitivity of an optical measurement to the local absorption change. ${ }^{6}$ By scanning the local absorber and measuring the signal change, a spatial sensitivity map can be obtained. In a previous publication, the authors first applied this approach to investigate the spatial sensitivity of AO measurements. ${ }^{19}$

The aim of this paper is to compare the sensitivity maps of three different detection techniques, namely the SSD, SR, and AO techniques. As mentioned above, the sensitivity maps were generated by scanning a local absorber in a homogeneous turbid medium and measuring the corresponding signal change. Experiments were performed in both transmission and reflection modes. The three techniques were compared in terms of the sensitivity in the ROI and the mean penetration depth in the reflection mode. The results shown here will be useful for developing tissue oxygenation monitors based on AO.

\section{Materials and Methods}

\subsection{Experiments}

\subsubsection{Phantoms}

The basic setup of the experiment is shown in Fig. 1(a). Two phantoms as shown in Fig. 1(b) and 2(c) were built, which contained Intralipid solution for transmission and reflection mode measurements. The Intralipid solution was diluted with deionized water to $1 \%$ volume ratio ${ }^{20,21}$ to achieve a transport scattering coefficient $\mu_{s}^{\prime}$ of $12 \mathrm{~cm}^{-1}$ (value typical of those found in tissues) and an absorption coefficient $\mu_{a}$ of $0.0235 \mathrm{~cm}^{-1}$ at an optical wavelength of $633 \mathrm{~nm}$. The two side walls of the phantoms were made of soft silicon rubber (ACC silicones MM228) with a Young's modulus of $0.62 \mathrm{MPa}$ to allow the propagation of the focused US waves into and out of the phantoms. The other walls were made of 3-mm thick clear glass. The local absorber had a dimension of $5 \times 5 \times 12 \mathrm{~mm}$ with $\mu_{a}=40.3 \mathrm{~cm}^{-1}$ and $\mu_{s}^{\prime}$ $=12 \mathrm{~cm}^{-1}$ at $780 \mathrm{~nm}$. The high absorption of the local absorber was to ensure photon path deletion and not intended to reflect a
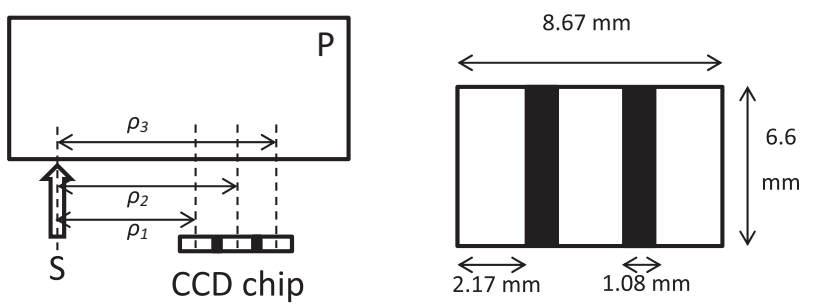

Fig. 2 The schematic of the segmentation of the CCD image area (1344 $\times 1024$ pixels) into three independent optical detectors where $\rho_{1}(26.8$ $\mathrm{mm}), \rho_{2}(30 \mathrm{~mm})$, and $\rho_{3}(33.2 \mathrm{~mm})$ are the distances between the source and the first, second, and third detectors, respectively. [P: phantom, S: optical source.] 
physiological condition. It was made of $10 \%$ polyvinyl alcohol (PVA) gel ${ }^{22}$ (Young's modulus of $\sim 20 \mathrm{kPa}$ at $20 \%$ strain) mixed with black polyurethane pigment (Tiranti, 410-520). The local absorber was held by a thin metallic rod inside the phantoms.

To assess the effect of the different acoustic properties between the local absorber and the Intralipid on the AO measurements, another local perturbation was made which we called the acoustic perturbation. The acoustic perturbation was made of the same materials and had the same dimension as the local absorber but with $\mu_{a}$ and $\mu_{s}$ similar to those of the Intralipid. The acoustic perturbation and the Intralipid together formed an optically homogeneous medium. In general, the PVA gel local absorber and acoustic perturbation have similar acoustic properties $^{23}$ (in terms of speed of sound and density) as the background Intralipid. ${ }^{24}$ Furthermore the PVA gel has a Poisson ratio of $0.499,{ }^{10}$ which implies that it is incompressible like the background water-based Intralipid solution.

\subsubsection{Acousto-optic detection system}

There are many types of AO detection systems, e.g., the speckle contrast system, ${ }^{25,26}$ the four-phase parallel detection system, ${ }^{27}$ and the photorefractive crystal system. ${ }^{28}$ The one used here is the digital autocorrelator system ${ }^{10,29}$ which measures the autocorrelation function of the AO signal.

The optical part of the AO system included an $808 \mathrm{~nm}$ single longitudinal mode laser (CrystaLaser, IRCL-100-808$\mathrm{S}$, coherence length $>10 \mathrm{~m}$ ), a single photon counter (Perkin Elmer, SPCM-AQR-14) and a digital correlator (Correlator.com, Flex02-01D). The laser was fiber-coupled to a $62.5 \mu \mathrm{m}$ multimode optical fiber to deliver light to the phantoms. The single photon counter was also fiber-coupled to a single mode $9 \mu \mathrm{m}$ optical fiber to detect light emerged from the phantoms. The digital correlator was connected to a computer and controlled by in-house LABVIEW software. For each position of the local absorber, an integration time of $20 \mathrm{~s}$ was used for the digital correlator to measure a normalized intensity autocorrelation function, $g_{x, y}^{(2)}(\tau)$ between the lag time of $20 \mathrm{~ns}$ and $15.36 \mu \mathrm{s}$ using the linear mode sampling with a sampling period of $20 \mathrm{~ns}$.

The US part of the AO system included an immersion US transducer with a center frequency of $0.66 \mathrm{MHz}$ (Precision Acoustics, PA304). It has a focal distance of $73 \mathrm{~mm}$, a focal zone width of $4.4 \mathrm{~mm}$, and a focal zone length of $29.4 \mathrm{~mm}$. The US transducer was operated in the $\mathrm{cw}$ mode to achieve a peak acoustic pressure of $181 \mathrm{kPa}$ at its peak focal region in the phantoms. At this rather low acoustic pressure, cavitation and excessive heating were unlikely. The US transducer was driven by a signal generator (Agilent Technologies, 33210A) through an RF amplifier (Electronics \& Innovation Ltd., A075). The configuration of the US transducer, optical source, and detector can be found in Figs. 1(b) and 1(c).

The US transducer was placed perpendicular to the optical source and detector as shown in Figs. 1(b) and 1(c). This configuration ensured that the superficial region of the phantom (the region close to the optical detector) would not be significantly affected by the US beam. We have shown in our previous work ${ }^{30}$ that such configuration for the AO measurement is feasible for a cylindrical geometry representing the limbs or the neck. This configuration has also been used in a clinical study to assess osteoporosis in the human wrist. ${ }^{31}$

\subsubsection{Optical detection systems}

The two optical detection systems were the SSD and SR systems. The same $808 \mathrm{~nm}$ laser employed in the AO system was also used here for both systems. For the SSD system, the photon counter and digital correlator as mentioned in Sec. 2.1.2 could also be used to collect intensity measurement in terms of photon counts per second $(\mathrm{Hz})$.

For the SR system, a 12-bit $1344 \times 1024(8.67 \times 6.60 \mathrm{~mm})$ CCD camera (Hamamatsu Orca 03G02) was used to measure the spatial distribution of the detected light from which the attenuation slope (SR) measurements could be calculated. The speckle size was adjusted by a lens system so that it matched the pixel size of the CCD chip $(6.45 \mu \mathrm{m})$. The exposure time was set to $5 \mathrm{~ms}$ to ensure adequate collection of light. It was found that outliners occasionally appeared on some of the CCD pixels due to noise. In order to remove them, five CCD images were taken for each position of the local absorber and one image free of outliers was chosen out of the five CCD images. The chosen image corresponded to the median of the five averaged values of the CCD images. More details will be given in Sec. 2.2.3 for calculating the attenuation slope based on this chosen CCD image. The configuration of the optical source and detector can be found in Figs. 1(b) and 1(c).

\subsubsection{Scanning}

A LABVIEW program was written to automatically control: i. the position of the local absorber within the liquid phantom via a 3-axis motorized translation stage (Zaber LSR150A), ii. the acquisition of the optical and AO measurements, and iii. the acquisition of the acoustic pressure measurements. The local absorber was positioned in the axial plane of the focused US and was scanned with a $1 \mathrm{~mm}$ resolution in the $x$ and $y$ directions. Ideally, for a $1 \mathrm{~mm}$ resolution, the size of the local absorber should be $1 \times 1 \mathrm{~mm}$ as well. However, a larger size $(5 \times 5 \mathrm{~mm})$ was necessary in this experiment to ensure adequate changes in the AO signal could be detected since the AO signal was very weak. The texture of the PVA gel was designed to be very soft in order to match the acoustic properties of the background medium as much as possible. A thin dimension of $1 \times 1 \mathrm{~mm}$ would have caused it to bend when submerged in the background medium because of its tendency to float. The total scanning area was $24 \times 24 \mathrm{~mm}$ (including scans at $0 \mathrm{~mm}$ ) for the transmission mode resulting in a total of 625 measurements, and $30 \times 20 \mathrm{~mm}$ for the reflection mode resulting in a total of 651 measurements, as shown in Figs. 1(b) and 1(c) respectively. In each set of scanning, a reference measurement was also taken without the local absorber inside the liquid phantom in order to calculate the sensitivity $J$ at location $(x, y)$ in the scanning area using Eq. (1).

$$
J(x, y)=\frac{M^{\mathrm{ref}}(x)-M(x, y)}{M^{\mathrm{ref}}(x)} \times 100 \%,
$$

where $M(x, y)$ is the measurement when the local absorber is at location $(x, y)$ and $M^{\mathrm{ref}}(x)$ is the reference measurement when the local absorber is removed from $(x, y)$ (out of the phantom) for the calculation of $J(x, y)$.

The reference measurement $M^{\text {ref }}(x)$ is also to account for the slow precipitation of the Intralipid solution over time besides for the calculation of $J(x, y)$. However, a reference measurement 
for every location $(x, y)$ would significantly increase the total measurement time that might result in significant changes in the optical properties of the Intralipid solution. Therefore the reference measurement $\mathrm{M}^{\mathrm{ref}}(x)$ was made only for each $x$ location of $J(x, y)$.

For each set of the experiment, a map of US pressure was also measured separately as a reference. It was obtained by scanning a needle hydrophone (1-mm diameter, Precision Acoustics HP1) with the same scanning area and resolution as above. The needle hydrophone was tilted at $15^{\circ}$ so that it could be accommodated inside the liquid phantoms in all positions during the scanning. However, since the needle hydrophone was tilted, the US pressure measurements were scaled. To resolve this problem, the US pressure measurements were re-scaled so that the US peak pressure was $181 \mathrm{kPa}$ in the maximum point in the US focus. This value of $181 \mathrm{kPa}$ was obtained by properly positioning the needle hydrophone in the US focus.

\subsubsection{Transmission and reflection modes}

For the transmission mode measurement as shown in Fig. 1(b), the US focus was located in the middle of the scanning area (12 $\mathrm{mm}$ from the boundary of the scanning area). The distance between the optical source and detector was $35 \mathrm{~mm}$. For the reflection mode measurement as shown in Fig. 1(c), four sets of measurements were taken for four different locations of the US focus (which were located around $y=10,15,20$, and $25 \mathrm{~mm}$ ) so that their sensitivity maps can be compared. The distance between the optical source and detector was $30 \mathrm{~mm}$.

\subsection{Post-Processing}

\subsubsection{AO system}

One widely adopted way of analyzing an AO signal is by considering its electric field autocorrelation function, $g_{x, y}^{(1)}(\tau) .{ }^{9,10,29}$ However, the digital correlator provided a slightly different measurement, i.e., the normalized intensity autocorrelation function, $g_{x, y}^{(2)}(\tau)$. Using the Siegert relationship, $g_{x, y}^{(1)}(\tau)$ can be derived from $g_{x, y}^{(2)}(\tau)$, i.e., $g_{x, y}^{(1)}(\tau)=\sqrt{\left[g_{x, y}^{(2)}(\tau)-1\right] / \beta}$, where $\beta$ is the coherence factor of the collection optics. ${ }^{32}$

It has been shown that $g_{x, y}^{(1)}(\tau)$ carries information about the proportion of the US modulated intensity, and the modulation depth (MD) of $g_{x, y}^{(1)}(\tau)$ defined in the following is one measure of the magnitude of the $\mathrm{AO}$ signal, ${ }^{9} \mathrm{MD}(x, y)$ $=\mathrm{FT}_{f_{a}}\left\{g_{x, y}^{(1)}(\tau)\right\} / \mathrm{FT}_{f_{0}}\left\{g_{x, y}^{(1)}(\tau)\right\}$, where $f_{0}$ and $f_{a}$ are the frequencies at $0 \mathrm{~Hz}$ and at the acoustic frequency $(0.66 \mathrm{MHz})$, respectively, and $\mathrm{FT}_{f}$ signifies the amplitude of the Fourier transform at frequency $f$. The MD has been described as the ratio of US modulated intensity to unmodulated intensity. ${ }^{9}$ The term sensitivity refers to the percentage change of the measurement once the local absorber has been inserted into the phantom. For the AO system, the measurement is $\operatorname{MD}(x, y)$ and the sensitivity is defined based on Eq. (1): $J_{\mathrm{AO}}(x, y)$ $=\left[\mathrm{MD}^{\mathrm{ref}}(x)-\mathrm{MD}(x, y)\right] / \mathrm{MD}^{\mathrm{ref}}(x) \times 100 \%$, where $\operatorname{MD}(x, y)$ is the MD measurement with the local absorber in the location $(x, y)$, and $\operatorname{MD}^{\text {ref }}(x)$ is the MD measurement without any local absorber in the turbid medium. In this study, one measurement of $\mathrm{MD}^{\text {ref }}(x)$ was taken for each position of $x$ for $J_{\mathrm{AO}}(x, y)$. These
$\operatorname{MD}^{\text {ref }}(x)$ measurements over all the $x$ positions were generally quite consistent with each other.

\subsubsection{SSD system}

As mentioned in Sec. 2.1.3, the photon counter and digital correlator also provided the intensity measurement in terms of photon counts per second $(\mathrm{Hz})$. Following a similar approach to Eq. (1), the sensitivity measure for the SSD system is defined as $J_{\mathrm{SSD}}(x, y)=\left[I_{\mathrm{SSD}}^{\mathrm{ref}}(x)-I_{\mathrm{SSD}}(x, y)\right] / I_{\mathrm{SSD}}^{\mathrm{ref}}(x) \times 100 \%$, where $I_{\mathrm{SSD}}(x, y)$ is the intensity measurement with the local absorber in the location $(x, y), I_{\mathrm{SSD}}^{\text {ref }}(x)$ is the reference measurement without any local absorber inside the medium and taken for each position of $x$ for $J_{\mathrm{SSD}}(x, y)$.

\subsubsection{SR system}

The SR system is based on the attenuation slope measurement, which is only valid in the reflection mode. Commercial systems such as the Hamamatsu NIRO 300 employ a few closely spaced optical detectors to measure the attenuation slope, ${ }^{4}$ i.e., three detectors each with a dimension of $8 \times 3 \mathrm{~mm}$ separated by 1 $\mathrm{mm}$. To calculate the attenuation slope from our CCD image, it was segmented into three detection areas as shown in Fig. 2 with the three detectors having a dimension of $6.6 \times 2.17 \mathrm{~mm}$ separated by $1.08 \mathrm{~mm}$. The distances from the center of each segment to the optical source are $\rho_{1}(26.8 \mathrm{~mm}), \rho_{2}(30 \mathrm{~mm})$, and $\rho_{3}(33.2 \mathrm{~mm})$. Each detector provided an intensity measurement which was converted into attenuation $A_{n}=-\log \left(I_{n}\right)$, where $I_{n}$ was the intensity of detector $n$ and $n=1,2$, and 3. A linear regression (least square) was then performed on $A_{n}$ using $\rho_{n}$ as the independent variable, where $\rho_{n}$ is the distance between the source and detector $n$. The slope of the regressed straight line was taken as the attenuation slope $\partial A / \partial \rho$.

Similar to Secs. 2.2.1-2.2.2, the sensitivity measure for the SR system is defined as $J_{\mathrm{SR}}(x, y)$ $=\left[\partial A^{\mathrm{ref}}(x) / \partial \rho-\partial A(x, y) / \partial \rho\right] /\left[\partial A^{\mathrm{ref}}(x) / \partial \rho\right] \times 100 \%$, where $\partial A(x, y) / \partial \rho$ is the attenuation slope measurement with the local absorber in the location $(x, y)$, and $\partial A^{\mathrm{ref}}(x) / \partial \rho$ is the reference measurement without any local absorber inside the medium and taken for each position of $x$ for $J_{\mathrm{SR}}(x, y)$.

\subsubsection{Mean sensitivity and penetration depth}

The spatial sensitivity map $J(x, y)$ provides a set of twodimensional data over the $x$ (in parallel to the superficial layer) and $y$ (perpendicular to the superficial layer or depth) directions. In this work, we also consider the mean sensitivity $\langle J(y)\rangle$ which is calculated as the averaged sensitivity values over the $x$ direction for each value of $y$.

Another useful way to compare the different sensing techniques in the reflection mode is to consider their mean penetration depths $\langle y\rangle$ which can be calculated as follows: ${ }^{33}\langle y\rangle$ $=\sum_{i} J\left(y_{i}\right) y_{i} / \sum_{i} J\left(y_{i}\right)$. With the SSD system in the reflection mode, some have defined the maximum penetration depth which is obtained by considering only the sensitivity values $J_{\mathrm{SSD}}(y)$ midway between the source and detector, and identifying the corresponding penetration depth $y$ when $J_{\mathrm{SSD}}(y)$ no longer stays above a predefined threshold. ${ }^{15,16,34}$ We prefer to use the mean penetration depth here because it is more representative of the whole layer at depth $y$. 


\section{Results}

The sensitivity measures $\left(J_{\mathrm{AO}}, J_{\mathrm{SSD}}\right.$, and $\left.J_{\mathrm{SR}}\right)$ are quite easy to interpret. A $10 \%$ (positive) sensitivity means that when the local absorber is inserted in the phantom, the measurement value decreases by $10 \%$ in comparison to the value before the insertion. On the contrary, a $-10 \%$ (negative) sensitivity means that the measurement value increases by $10 \%$ with the insertion of the local absorber. For $J_{\mathrm{SR}}$, sensitivity above $100 \%$ has been observed when the local absorber is very close to the detectors. This sensitivity over $100 \%$ corresponds to the situation when the attenuation slope and the reference measurement having an opposite sign in their values. In order to make a comparison easier, the color scales of all the spatial sensitivity maps here have been fixed between $0 \%$ and $100 \%$. However, it also means that negative values and values over $100 \%$ may not be obvious in the figures but these atypical values will be pointed out in the text.

As mentioned in Sec. 1, the ROI is often a region beneath the surface. As will be shown in Secs. 3.1-3.4, ROIs between 8.6 and $25 \mathrm{~mm}$ away from the optical detector have been defined in both the transmission and reflection modes. In the AO measurement, focused US was used to increase the ROI's sensitivity by positioning the focused US within the ROI. The ROI coincided with the $-3 \mathrm{~dB}$ point of the peak pressure of the focused US.

\subsection{Local Acoustic Perturbation}

It is known that $\mathrm{AO}$ measurements are affected by both optical and acoustic properties of the medium. ${ }^{10}$ An experiment was performed with the local acoustic perturbation as mentioned in Sec. 2.1.1 which had the same optical properties as the background Intralipid. The resulting spatial sensitivity map of the optical measurement $J_{\mathrm{SSD}}(x, y)$ in Fig. 3(c) does not show a substantial change confirming that the medium with the local acoustic perturbation inside is optically homogeneous. As for the AO measurements, $J_{\mathrm{AO}}(x, y)$ and its mean value as depicted in Figs. 3(b) and 3(d) show a small reduction in sensitivity values $(<5 \%)$ in the vicinity of the US focus. [Referring to Eq. (1), a reduction in sensitivity value corresponds to an increase in MD when the local acoustic perturbation is inserted into the background Intralipid.] Although the values are small, it indicates that the AO measurements are slightly influenced by the acoustic properties of the local perturbation. In Secs. 3.2-3.4, the sensitivity values increase by more than $40 \%$ in the US focus (corresponding to a reduction in MD) when the local absorber is used, showing that the optical property (absorption coefficient) of the local absorber is the dominant factor of the sensitivity rather than its acoustic properties.

\subsection{Transmission Mode}

Figure 4 shows the results for the transmission mode including (a) the US peak pressure map, the spatial sensitivity maps for (b) the $\mathrm{AO}$ measurement $J_{\mathrm{AO}}(x, y)$, (c) the SSD measurement $J_{\mathrm{SSD}}(x, y)$, and (d) the mean sensitivity over different depths. The coordinates of the optical source are near to $(0,5.5)$ and those of the detector are near to $(0,29.5)$ in Figs. 4(b) and 4(c). The ROI is defined as the region between 16 and $20 \mathrm{~mm}$ away from the detector plane, approximately in the middle of the (a) US Peak Pressure $(\mathrm{kPa})$

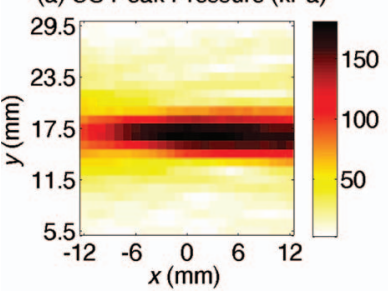

(c) $J_{\text {SSD }}(\%)$

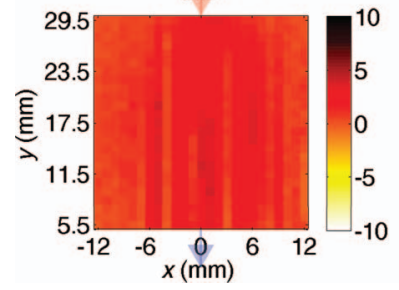

(b) $\mathrm{J}_{\mathrm{AO}}(\%)$

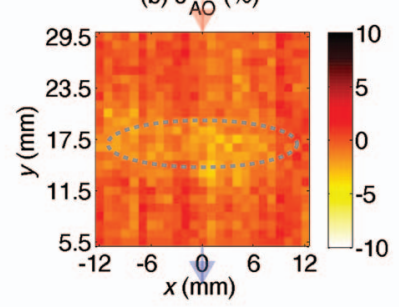

(d) $<$ J $(y)>(\%)$

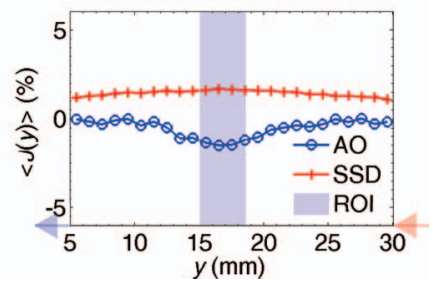

Fig. 3 Transmission mode with a local acoustic perturbation with low $\mu_{a}$ : (a) the US peak pressure map with the US focus within the ROI, (b) the spatial sensitivity map of the $\mathrm{AO}$ measurement $J_{\mathrm{AO}}(x, y)$, the dotted ellipse marks the ROI, (c) the spatial sensitivity map of the SSD measurement $J_{S S D}(x, y)$, (d) the mean sensitivities of the $\mathrm{AO}$ and SSD measurements, i.e., $\left\langle J_{\mathrm{AO}}(y)\right\rangle$ and $\left\langle J_{\mathrm{SSD}}(y)\right\rangle$. The red and blue shaded arrows mark the approximate locations of the optical source $(x=0 \mathrm{~mm}$ and $y=35 \mathrm{~mm}$ ) and detector $(x=0 \mathrm{~mm}$ and $y=0 \mathrm{~mm})$, respectively. Note the color scaling is much smaller than those in Figs. 4 and 5.

turbid medium. The focused US has been positioned to the ROI as shown in Fig. 4(a).

For the SSD measurement as shown in Fig. 4(c), the regions with the relative peak sensitivity are near the source and detector (superficial region), rather than in the vicinity of the ROI. On the contrary for the AO measurement as shown in Fig. 4(b), the region with the relative peak sensitivity coincides with the ROI.
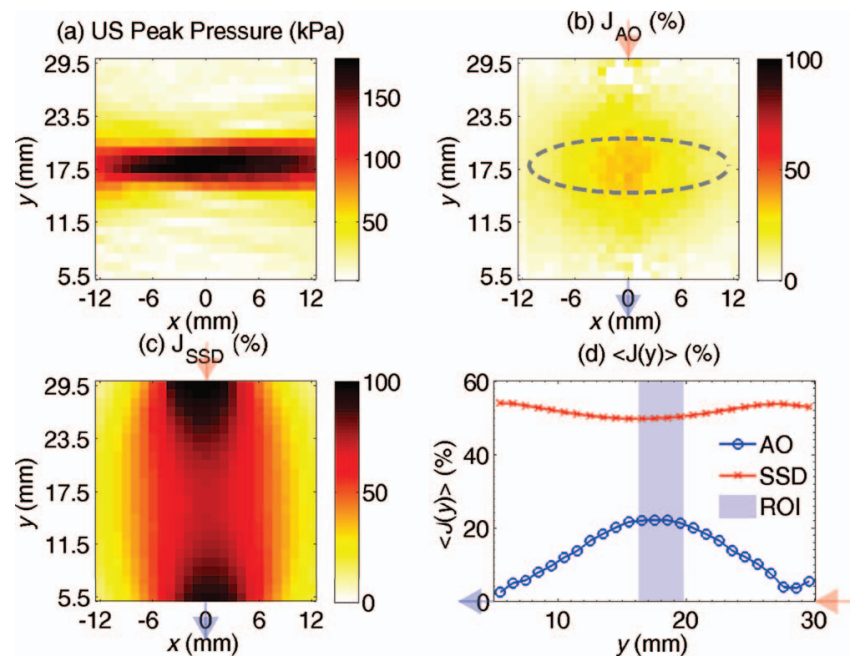

Fig. 4 Transmission mode with a local absorber with high $\mu_{a}$ : (a) the US peak pressure map with the US focus within the ROI, (b) the spatial sensitivity map of the $\mathrm{AO}$ measurement $J_{\mathrm{AO}}(x, y)$, the dotted ellipse marks the ROI, (c) the spatial sensitivity map of the SSD measurement $J_{\mathrm{SSD}}(x, y)$, (d) the mean sensitivities of the AO and SSD measurements, i.e., $\left\langle J_{\mathrm{AO}}(y)\right\rangle$ and $\left\langle J_{\mathrm{SSD}}(y)\right\rangle$. The red and blue shaded arrows mark the approximate locations of the optical source $(x=0 \mathrm{~mm}$ and $y=35 \mathrm{~mm})$ and detector $(x=0 \mathrm{~mm}$ and $y=0 \mathrm{~mm})$, respectively. 
The focused US successfully shifts the most sensitive point to the ROI.

Figure 4(d) shows the mean sensitivity $\langle J(y)\rangle$ at different depths along the $y$ direction (away from the detector plane). Once again, it is evident from this figure that $\left\langle J_{\mathrm{AO}}(y)\right\rangle$ has the relative peak value in the ROI (shaded area) while $\left\langle J_{\mathrm{SSD}}(y)\right\rangle$ has its relative peak value near to the detector. In terms of the absolute value of the sensitivity, however, $\left\langle J_{\mathrm{SSD}}(y)\right\rangle$ in general has higher absolute values than $\left\langle J_{\mathrm{AO}}(y)\right\rangle$ throughout the sensitivity map.

It is worth noting that when the local absorber is very near the optical source around location $(0,29)$ in Fig. $4(\mathrm{~b}), J_{\mathrm{AO}}$ becomes negative which means that the $\mathrm{AO}$ measurement, i.e., $\mathrm{MD}$, is in fact increased in value with the insertion of the local absorber. To understand this phenomenon, we need to consider again the AO measurement $\mathrm{MD}$, which is the ratio of ultrasound modulated intensity to unmodulated intensity. When the local absorber is near to the US focus, more US modulated intensity will be absorbed and therefore a smaller MD. However, when the local absorber is near the source, more unmodulated intensity will be absorbed in comparison to the modulated intensity resulting in an increased MD.

\subsection{Reflection Mode}

Figure 5 shows the results for the reflection mode including two ROIs, i.e., ROI A and ROI B. The coordinates of the optical

(a) US Peak Pressure A ( $\mathrm{kPa})$

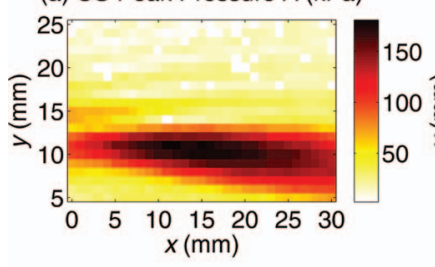

(c) $\mathrm{J}_{\mathrm{AO}} \mathrm{A}(\%)$

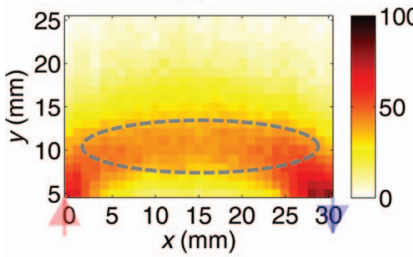

(e) $\mathrm{J}_{S S D}(\%)$
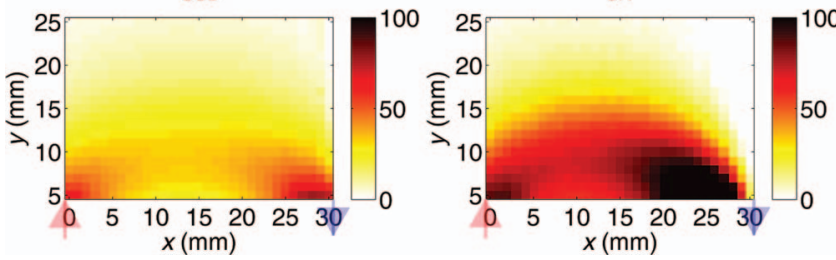

Fig. 5 Reflection mode with a local absorber with high $\mu_{a}$ : the US peak pressure maps with the focus within (a) the ROI A and (b) the $\mathrm{ROI} \mathrm{B}$. The spatial sensitivity maps of the $\mathrm{AO}$ measurements with the US focus in (c) ROI A (the dotted ellipse), $J_{\mathrm{AO}} A(x, y)$, and (d) ROI B (the dotted ellipse), $J_{\mathrm{AO}} B(x, y)$. The spatial sensitivity maps of (e) the SSD measurements, $J_{\mathrm{SSD}} B(x, y)$ and (f) the SR measurements, $J_{\mathrm{SR}}(x, y)$. The red and blue shaded arrows mark the approximate locations of the optical source $(x=0 \mathrm{~mm}$ and $y=0 \mathrm{~mm})$ and detector $(x=30 \mathrm{~mm}$ and $y=0 \mathrm{~mm})$, respectively. source were near to $(0,5)$ and those of the detector are near to $(0,30)$ in Figs. 5(c)-5(f). The ROI A is defined as the region between 8.6 and $12.7 \mathrm{~mm}$ away from the source-detector plane, whereas ROI B is the region between 19.4 and $23.0 \mathrm{~mm}$. The focused US is positioned in such a way that it coincides with the two ROIs in two separate experiments as shown in Figs. 5(a) and 5(b). Figures 5(c) and 5(d) depict the sensitivity maps of the AO measurements when the focused US is within ROI A, i.e., $J_{\mathrm{AO}} A$ and within ROI B, i.e., $J_{\mathrm{AO}} B$. Figures 5(e) and 5(f) show the sensitivity maps for the SSD and SR measurements, i.e., $J_{\mathrm{SSD}}$ and $J_{\mathrm{SR}}$.

Figures 5(c)-5(f) show some common features for all the sensitivity maps. First, the banana shaped distribution is evident. Second, the relative peak sensitivity can be found near to the optical source and detector.

Figure 6 shows the mean sensitivity $\langle J(y)\rangle$ at different depths along the $y$ direction (away from the source-detector plane). The two ROIs are shown as the two shaded areas. It can be seen that $\left\langle J_{\mathrm{AO}} A(y)\right\rangle$ has the relative peak value in ROI A while $\left\langle J_{\mathrm{AO}} B(y)\right\rangle$, $\left\langle J_{\mathrm{SSD}}(y)\right\rangle$, and $\left\langle J_{\mathrm{SR}}(y)\right\rangle$ have the relative peak values outside ROI A. This shows that the focused US in Fig. 5(a) successfully shifts the peak mean sensitivity point of the $\mathrm{AO}$ measurement toward ROI A.

However, when the focused US is moved to ROI B as shown in Fig. 5(b), the peak value of $\left\langle J_{\mathrm{AO}} B(y)\right\rangle$ is found at a depth of around $y=15 \mathrm{~mm}$ which is outside ROI B. This shows that although a focused US at a greater depth can lead to a higher sensitivity of an AO measurement toward a greater depth, the peak sensitivity point does not always coincide with the actual location of the focused US. Despite this fact, the highest mean sensitivity in ROI B is still the one from $\left\langle J_{\mathrm{AO}} B(y)\right\rangle$ in comparison to $\left\langle J_{\mathrm{AO}} A(y)\right\rangle,\left\langle J_{\mathrm{SSD}}(y)\right\rangle$, and $\left\langle J_{\mathrm{SR}}(y)\right\rangle$ in Fig. 6.

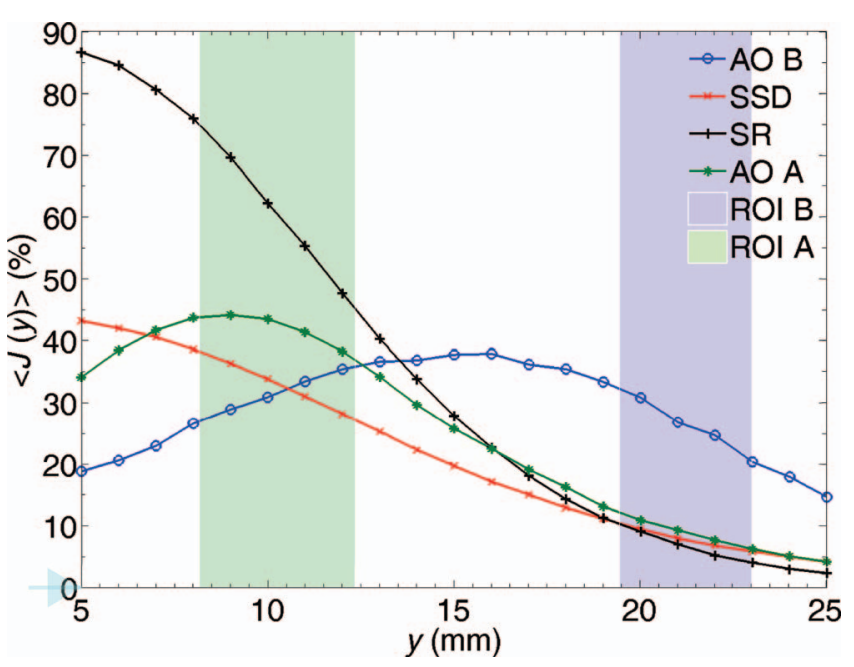

Fig. 6 Reflection mode with a local absorber with high $\mu_{\mathrm{a}}$ : The mean sensitivities of the $\mathrm{AO}$ measurements with the US focus within ROI A $\left\langle J_{\mathrm{AO}} A(y)\right\rangle$ (symbol "**", green curve) and within ROI B $\left.\bigcup_{\mathrm{AO}} B(y)\right\rangle$ (symbol " $\mathrm{o}$ ", blue curve). The mean sensitivities of the SSD measurements $\left\langle J_{S S D}(y)\right\rangle$ (symbol " $\mathrm{x}^{\prime}$, red curve) and SR measurements $\left\langle J_{\mathrm{SR}}(y)\right\rangle$ (symbol "+", black curve). The ROls A (green) and B (blue) are shown as shaded areas. The $y$-axis can be regarded as the penetration depth. The aqua shaded arrow marks the approximate location of the optical source and detector plane $(y=0 \mathrm{~mm})$. 
Table 1 Reflection mode: mean penetration path (in the $y$-axis) and the comparison of the mean sensitivity between the superficial region and the two ROls, which are located around $y=10 \mathrm{~mm}$ (ROI A) and $y=21 \mathrm{~mm}$ (ROI B). The sensitivities of the two ROls shown here are the maximum values within the ROIs.

\begin{tabular}{|c|c|c|c|c|}
\hline \multirow[b]{2}{*}{$\begin{array}{l}\text { Sensing } \\
\text { technique }\end{array}$} & \multirow[b]{2}{*}{$\begin{array}{c}\text { Mean penetration } \\
\text { depth in the } \\
y \text {-axis }(\mathrm{mm})\end{array}$} & \multirow[b]{2}{*}{$\begin{array}{l}\text { Mean sensitivity } \\
\text { in the superficial } \\
\text { region }(y=5 \mathrm{~mm})\end{array}$} & \multicolumn{2}{|c|}{ Maximum mean sensitivity } \\
\hline & & & $\begin{array}{c}\text { In ROI A } \\
\text { (higher mean } \\
\text { sensitivity in ROI A?) }\end{array}$ & $\begin{array}{c}\text { In ROI B } \\
\text { (higher mean } \\
\text { sensitivity in ROI } \mathrm{B} \text { ?) }\end{array}$ \\
\hline Single source and detector, $\left\langle J_{S S D}\right\rangle$ & 11.4 & $43 \%$ & $31 \%(\mathrm{NO})$ & $8 \%(\mathrm{NO})$ \\
\hline Spatially resolved, $\left\langle J_{S R}\right\rangle$ & 10.2 & $87 \%$ & $55 \%(\mathrm{NO})$ & $7 \%(\mathrm{NO})$ \\
\hline Acousto-optics, $\left\langle J_{A O} A\right\rangle$, Focused US in ROI A & 11.8 & $34 \%$ & $41 \%$ (YES) & $9 \%(N O)$ \\
\hline Acousto-optics, $\left\langle J_{A O} B\right\rangle$, Focused US in ROI B & 14.8 & $19 \%$ & $33 \%$ (YES) & $27 \%$ (YES) \\
\hline
\end{tabular}

A summary of the mean sensitivities in the superficial region (at a depth of $y=5 \mathrm{~mm}$ ) and the two ROIs is shown in Table 1 . It shows that the two optical techniques $\left\langle J_{\mathrm{SSD}}(y)\right\rangle$ and $\left\langle J_{\mathrm{SR}}(y)\right\rangle$ have higher values ("+" and " $x$ " curves) in the superficial region than in the two ROIs. As for the AO technique, when the focused US coincides with ROI A, the $\left\langle J_{\mathrm{AO}} A(y)\right\rangle$ ("**" curve) in ROI A is higher than that in the superficial region. With the focused US at this location, the $\left\langle J_{\mathrm{AO}} A(y)\right\rangle$ in ROI $\mathrm{B}$ is lower than that in the superficial region. When the focused US coincided with ROI B, the $\left\langle J_{\mathrm{AO}} B(y)\right\rangle$ ("o" curve) in both ROI A and ROI $\mathrm{B}$ are higher than that in the superficial region.

The mean penetration depths of different sensing techniques are shown in Table 1. The AO technique with the focused US in ROI B has the greatest penetration depth of $14.8 \mathrm{~mm}$, whereas the SR technique has the lowest penetration depth of $10.2 \mathrm{~mm}$.

As in the transmission mode, it is noted that certain $J_{\mathrm{AO}} B$ values in Fig. 5(d) are negative which occurs when the local absorber is located midway between the source and detector in the superficial region. As already discussed in Sec. 3.1, this is due to the fact that more unmodulated intensity is absorbed by the local absorber in this particular region, resulting in an increase in the MD.

\subsection{Acousto-Optic Measurements With the Focused Ultrasound at Various Depths}

Figure 7 shows the mean sensitivities of the AO measurements with the focused US at four different locations, corresponding to four ROIs. Table 2 shows the comparison of the relative peak sensitivities, their locations, and the mean penetration depths. It can be seen that as the focused US is moved deeper, the relative peak value of $\left\langle J_{\mathrm{AO}}(y)\right\rangle$ becomes smaller and its location is also deeper. Except ROI A, the relative peak values of $\left\langle J_{\mathrm{AO}}(y)\right\rangle$ is not within the US focus at ROIs B, C, and D.

\section{Discussions}

\subsection{Relative Sensitivity Versus Absolute Sensitivity}

In the transmission mode as shown in Fig. 4, the peak relative sensitivity of the optical SSD technique is in the superficial region, whereas that of the AO technique is inside the ROI. The $\mathrm{AO}$ technique successfully produces a measurement, which is most sensitive to the ROI. However, one can also see that the absolute sensitivity of the SSD technique is in fact always higher than that of the AO technique, e.g., the SSD mean sensitivity curve is above the AO mean sensitivity curve at all depths in Fig. 4(d). This means that in the absence of any spurious absorption changes outside the ROI, the SSD technique is in fact more sensitive to absorption changes within the ROI than the AO technique.

Similarly for the reflection mode, Figs. 5 and 6 show that the peak relative sensitivities of the optical SSD and SR techniques are in the superficial region whereas that of the AO technique (with US focus in ROI A) is inside the ROI. However, the SR technique has the highest absolute sensitivity in ROI A despite the fact it has even higher sensitivity in the superficial region.

By comparing all the mean sensitivity curves in Fig. 6, we conclude that for a ROI deeper than $14 \mathrm{~mm}$ [the crossover point between the $\left\langle J_{\mathrm{AO}} B(y)\right\rangle$ and $\left\langle J_{\mathrm{SR}}(y)\right\rangle$ curves], the AO technique has a higher absolute sensitivity over all other optical techniques (SSD and SR). In the case of ROI B which is located $21 \mathrm{~mm}$

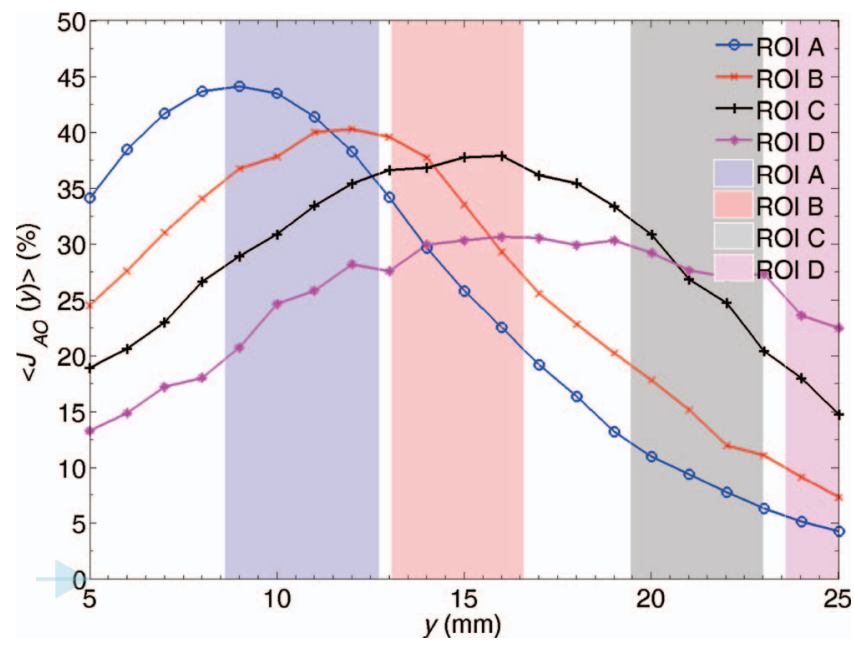

Fig. 7 Reflection mode with a local absorber with high $\mu_{a}$ : The mean sensitivities of the $\mathrm{AO}$ measurements with the US focus within ROI A $\left\langle J_{\mathrm{AO}} A(y)\right\rangle$, ROI B $\left\langle J_{\mathrm{AO}} B(y)\right\rangle$, ROI C $\left\langle J_{\mathrm{AO}} C(y)\right\rangle$, and ROI D $\left\langle J_{\mathrm{AO}} D(y)\right\rangle$. The shaded areas correspond to the four ROls. The $y$-axis can be regarded as the penetration depth. The aqua shaded arrow marks the approximate location of the optical source and detector plane $(y=0 \mathrm{~mm})$. 
Table 2 Reflection mode: comparison of the mean relative peak sensitivities, their locations, and the mean penetration depths of the four $\mathrm{AO}$ measurements. The locations of the mean relative peak sensitivities were inside ROI A but outside ROIs B, C, and D.

\begin{tabular}{lccc}
\hline $\begin{array}{l}\text { ROI } \\
\text { (position in } y \text {-axis })\end{array}$ & $\begin{array}{c}\text { Relative peak } \\
\text { sensitivity } \\
(\mathrm{mm})\end{array}$ & $\begin{array}{c}\text { Location in the } y \text {-axis of the } \\
\text { relative peak sensitivity } \\
(\mathrm{mm})\end{array}$ & $\begin{array}{c}\text { Mean penetration } \\
\text { depth in the } \\
y \text {-axis }(\mathrm{mm})\end{array}$ \\
\hline A (8.6 to 12.7) & $44.1 \%$ & 9 & 11.8 \\
B (13.1 to 16.6$)$ & $40.2 \%$ & 12 & 13.1 \\
C (19.4 to 23.0$)$ & $37.9 \%$ & 16 & 14.8 \\
D (23.6 to 25$)$ & $30.6 \%$ & 16 & 15.8 \\
\hline
\end{tabular}

deep from the superficial layer, the absolute mean sensitivity of the $\mathrm{AO}$ technique is more than twice those of other techniques.

\subsection{Relative Peak Sensitivity, Penetration Depth, and the Location of the Ultrasound Focus}

Figure 6(d) also shows that the relative peak sensitivity does not always coincide with the focused US. This finding is observed when the focused US is positioned inside ROI B and yet the peak sensitivity of the $\mathrm{AO}$ technique is not within it. On the other hand, Fig. 6(c) shows that the peak sensitivity occurs within the focused US when it is located in ROI A. Figure 6(e) shows that ROI A has a much higher optical spatial sensitivity $\left(J_{S S D}\right)$ than ROI B. Therefore the location of the peak sensitivity of the AO measurement depends on the optical spatial sensitivity in the focused US region.

One way to move the relative peak sensitivity of the AO technique inside ROI B would be to increase the optical sourcedetector spacing. Using the SSD system in the reflection mode, it has been shown both experimentally ${ }^{15,16}$ and by simulations s $^{7,34}$ that the penetration depth can be increased by increasing the optical source-detector spacing. (Although the optical spatial sensitivity $J_{\mathrm{SSD}}$ may change with the optical source-detector spacing, the peak relative sensitivity is likely to stay in the superficial region.) Therefore, increased optical source-detector spacing will also lead to a higher optical spatial sensitivity in the focused US region and therefore shifting the relative peak sensitivity of the AO technique deeper into the ROI B.

In comparison to all other techniques, the $\mathrm{AO}$ technique still has the highest absolute sensitivity within ROI B as evident in Fig. 6. The results in Fig. 7 and Table 2 further confirm that the relative peak sensitivity is not always within the US focus region. These results have significant implications for both $\mathrm{AO}$ sensing and imaging applications.

\subsection{Influence of the Acoustic Properties}

The sensitivity maps shown in Figs. 4(b), 5(c), and 5(d) account for both optical and acoustic property changes in the background medium. We have shown in Fig. 3(b) that the sensitivity changes due to acoustic property change are small $(<5 \%)$ and have negative values (increased MD), while in Figs. 4 and 5 the sensitivity changes due to both acoustic and optical property changes are much higher ( $>40 \%)$ and have positive value (decreased MD). One possible approach to minimize the effect of the acoustic property change in Fig. 4(b) would be to subtract it from Fig. 3(b). We, however, choose to present the results with less post-processing so that readers can see the original data for themselves. It is also important to realize that the AO measurement (amplitude of MD) is also dependent on the acoustic properties of the background medium (Intralipid in our case) which attenuate the US, and therefore limit its intensity in the focus and affect the amplitude of the MD.

\subsection{Comparison With Computational Results}

We have developed a Monte Carlo model for the calculation of the spatial sensitivity maps of the $\mathrm{AO}$ and optical detection schemes. ${ }^{35}$ Interested readers are referred to this paper for details. The main features of the computed spatial sensitivity maps are very similar to the experimental ones shown here. For example, in both the experimental and computational results, the relative peak sensitivities of the optical method (SSD) are near to the superficial region, while those of the AO method are near to the US focused region. These characteristics apply to both transmission and reflection modes. Also, in the reflection mode, the computed sensitive values of the AO method are negative in the region near to the surface and between the optical source and detector, which again agree with the experimental results here.

In the Monte Carlo simulations, a local absorber with a dimension of $1 \times 1 \mathrm{~mm}$ has been used, rather than a dimension of $5 \times 5 \mathrm{~mm}$ as in the experiments here. The similarity of results in both cases suggests that a local absorber with a larger size does not affect the experimental results considerably.

\subsection{Other Acousto-Optical Techniques}

Following the discussion of the low absolute sensitivity of the AO technique in certain situations, e.g., in transmission mode, it can in fact be improved by various means. For instance, with the existing AO system one straightforward way is to use a stronger focused US which will increase the particle displacement in the US focus and therefore the MD. ${ }^{36}$ Various AO imaging systems based on photorefractive crystal, ${ }^{28}$ spectral hole burning, ${ }^{37}$ and acoustic radiation force ${ }^{38,39}$ have also been developed to enhance the AO signals and can potentially produce higher absolute sensitivity.

\subsection{Other NIRS Techniques}

In this paper, we have only considered two NIRS techniques, i.e., the SSD and SR techniques, which are commonly used 
in commercial NIRS clinical monitors. There are in fact other commercial NIRS monitors, which exploit a multiple detector scheme such as the INVOS Cerebral Oximeter (Ref. 40) from Somanetics Co., and the FORE-SIGHT Cerebral Oximeter (Ref. 41) from CAS Medical Systems, Inc. In comparison with the SR system discussed here in which multiple detectors are placed very close to each other (millimeter spacing), these NIRS monitors have the multiple detectors more widely spaced (centimeter spacing). Other NIRS systems do not employ the $\mathrm{cw}$ laser source at all, including the phase resolved spectroscopy system, e.g., the OxiplexTS ${ }^{\mathrm{TM}}$ tissue oximeter ${ }^{42}$ from ISS Inc. and the time resolved spectroscopy system, e.g., the TRS system ${ }^{43}$ from Hamamatsu Photonics KK. In particular, the time resolved spectroscopy system has been shown to have a higher sensitivity toward the deeper layer in comparison to the SSD system. ${ }^{44}$

\subsection{Oxygenation Measurement}

In this work, we only consider the basic measurements in terms of intensity (SSD), attenuation slope (SR), and the modulation depth (AO). It is necessary to convert these basic measurements into the absorption coefficients $\mu_{a}$ first before one can apply the Beer-Lambert law to convert $\mu_{a}$ (measured in multiple wavelengths) into concentrations of oxy- and deoxyhaemoglobin for clinical monitoring. For the SSD and SR systems, this can be done by using the modified Beer-Lambert law ${ }^{45}$ and a solution of the diffusion equation under specific boundary conditions,${ }^{4}$ respectively. As for the AO system, a thorough theory that can convert the modulation depth into $\mu_{a}$ is still under development although early success has been reported based on approximations. ${ }^{11,13,14}$

\section{Conclusion}

In tissue oxygenation monitoring, the ROI is often beneath a superficial layer. Therefore, a good tissue oxygenation monitor should have a higher sensitivity to the deeper region than to the superficial region. However, many existing NIRS systems have the highest sensitivities in the superficial layer, rather than in the ROI. Through absorption perturbation experiments, we have shown that generally the AO technique can increase the sensitivity in a deeper region by incorporating a focused US with NIRS. In the reflection mode with a source-detector spacing of $30 \mathrm{~mm}$, the mean penetration depth of the AO technique was $14.8 \mathrm{~mm}$, whereas that of the optical SSD technique was 11.4 $\mathrm{mm}$ in one realistic scenario. This shows the potential of the AO technique for providing a more regional measurement for tissue oxygenation monitoring.

\section{Acknowledgments}

This research was funded by the Engineering and Physical Sciences Research Council (Grant Code EP/G005036/1).

\section{References}

1. M. Wolf, M. Ferrari, and V. Quaresima, "Progress of near-infrared spectroscopy and topography for brain and muscle clinical applications," J. Biomed. Opt. 12(6), 062104 (2007).

2. S. Feng, F. A. Zeng, and B. Chance, "Photon migration in the presence of a single defect: A perturbation analysis," Appl. Opt. 34(19), 3826-3837 (1995).
3. M. J. Hofmann, M. J. Herrmann, I. Dan, H. Obrig, M. Conrad, L. Kuchinke, A. M. Jacobs, and A. J. Fallgatter, "Differential activation of frontal and parietal regions during visual word recognition: An optical topography study," NeuroImage 40(3), 1340-1349 (2008).

4. S. Suzuki, S. Takasaki, T. Ozaki, and Y. Kobayashi, "A tissue oxygenation monitor using NIR spatially resolved spectroscopy," Proc. SPIE 3597, 582-592 (1999).

5. M. Buchheit, P. B. Laursen, and S. Ahmaidi, "Effect of prior exercise on pulmonary $\mathrm{O} 2$ uptake and estimated muscle capillary blood flow kinetics during moderate-intensity field running in men," J. Appl. Physiol. 107(2), 460-470 (2009).

6. S. R. Arridge, "Photon-measurement density functions. Part I: Analytical forms," Appl. Opt. 34(31), 7395-7409 (1995).

7. E. Okada and D. T. Delpy, "Near-infrared light propagation in an adult head model. I. Modeling of low-level scattering in the cerebrospinal fluid layer," Appl. Opt. 42(16), 2906-2914 (2003).

8. D. A. Boas and A. M. Dale, "Simulation study of magnetic resonance imaging-guided cortically constrained diffuse optical tomography of human brain function," Appl. Opt. 44(10), 1957-1968 (2005).

9. L. V. Wang, "Mechanisms of ultrasonic modulation of multiply scattered coherent light: An analytic model," Phys. Rev. Lett. 87(4), 043903 (2001).

10. C. U. Devi, R. S. Chandran, R. M. Vasu, and A. K. Sood, "Detection of optical and mechanical property inhomogeneities in tissue mimicking phantoms using an ultrasound assisted optical probe," J. Biomed. Opt. 13(6), 064025 (2008).

11. C. Kim and L. V. Wang, "Multi-optical-wavelength ultrasoundmodulated optical tomography: A phantom study," Opt. Lett. 32(16), 2285-2287 (2007).

12. S. R. Kothapalli, S. Sakadzic, C. Kim, and L. V. Wang, "Imaging optically scattering objects with ultrasound-modulated optical tomography," Opt. Lett. 32(16), 2351-2353 (2007).

13. A. Bratchenia, R. Molenaar, T. van Leeuwen, and R. P. Kooyman, "Millimeter-resolution acousto-optic quantitative imaging in a tissue model system," J. Biomed. Opt. 14(3), 034031 (2009).

14. A. Bratchenia, R. Molenaar, T. G. Van Leeuwen, and R. P. H. Kooyman, "Acousto-optic-assisted diffuse optical tomography," Opt. Lett. 36(9), 1539-1541 (2011).

15. T. Vaithianathan, I. D. C. Tullis, N. Everdell, T. Leung, A. Gibson, J. Meek, and D. T. Delpy, "Design of a portable near infrared system for topographic imaging of the brain in babies," Rev. Sci. Instrum. 75(10 I), 3276-3283 (2004).

16. A. V. Patil, J. Safaie, H. A. Moghaddam, F. Wallois, and R. Grebe, "Experimental investigation of NIRS spatial sensitivity," Biomed. Opt. Express 2(6), 1478-1493 (2011).

17. N. C. Bruce, "Experimental study of the effect of absorbing and transmitting inclusions in highly scattering media," Appl. Opt. 33(28), 66926698 (1994)

18. S. Fantini, M. A. Franceschini, S. A. Walker, J. S. Maier, and E. Gratton, "Photon path distributions in turbid media: Applications for imaging," Proc. SPIE 2389, 340-349 (1995).

19. S. Gunadi and T. S. Leung, "Regional sensitivity comparison between optical and acousto-optic sensing," Proc. SPIE 7899, 78992Q (2011).

20. H. J. van Staveren, C. J. M. Moes, J. van Marie, S. A. Prahl, and M. J. C. van Gemert, "Light scattering in Intralipid-10\% in the wavelength range of 400-1100 nm," Appl. Opt. 30(31), 4507-4514 (1991).

21. S. T. Flock, S. L. Jacques, B. C. Wilson, W. M. Star, and M. J. C. Van Gemert, "Optical properties of intralipid: A phantom medium for light propagation studies," Laser. Surg. Med. 12(5), 510-519 (1992).

22. B. Price, A. Gibson, and G. Royle, "Dual-modality imaging of a compressible breast phantom with realistic optical and x-ray properties," Proc. SPIE 7622, 762207 (2010).

23. K. Zell, J. I. Sperl, M. W. Vogel, R. Niessner, and C. Haisch, "Acoustical properties of selected tissue phantom materials for ultrasound imaging," Phys. Med. Biol. 52(20), N475-N484 (2007).

24. J. Laufer, E. Zhang, and P. Beard, "Evaluation of absorbing chromophores used in tissue phantoms for quantitative photoacoustic spectroscopy and imaging," IEEE J. Sel. Top. Quantum Electron. 16(3), 600-607 (2010). 
25. J. Li, G. Ku, and L. V. Wang, "Ultrasound-modulated optical tomography of biological tissue by use of contrast of laser speckles," Appl. Opt. 41(28), 6030-6035 (2002).

26. R. Li, L. Song, D. S. Elson, and M. X. Tang, "Parallel detection of amplitude-modulated, ultrasound-modulated optical signals," Opt. Lett. 35(15), 2633-2635 (2010).

27. S. Leveque-Fort, "Three-dimensional acousto-optic imaging in biological tissues with parallel signal processing," Appl. Opt. 40(7), 1029-1036 (2001).

28. T. W. Murray, L. Sui, G. Maguluri, R. A. Roy, A. Nieva, F. Blonigen, and C. A. DiMarzio, "Detection of ultrasound-modulated photons in diffuse media using the photorefractive effect," Opt. Lett. 29(21), 2509-2511 (2004).

29. W. Leutz and G. Maret, "Ultrasonic modulation of multiply scatteredlight," Physica B 204(1-4), 14-19 (1995).

30. S. Gunadi, S. Powell, C. E. Elwell, and T. S. Leung, "Optimization of the acousto-optic signal detection in cylindrical geometry," Proc. SPIE 7564, 756431 (2010).

31. A. Lev, E. Rubanov, B. Sfez, S. Shany, and A. J. Foldes, "Ultrasoundmodulated light tomography assessment of osteoporosis," Opt. Lett. 30(13), 1692-1694 (2005).

32. S. E. Skipetrov and I. V. Meglinskiǐ, "Diffusing-wave spectroscopy in randomly inhomogeneous media with spatially localized scatterer flows," J. Exp. Theor. Phys. 86(4), 661-665 (1998).

33. S. Del Bianco, F. Martelli, and G. Zaccanti, "Penetration depth of light re-emitted by a diffusive medium: Theoretical and experimental investigation," Phys. Med. Biol. 47(23), 4131-4144 (2002).

34. T. Correia, A. Gibson, and J. Hebden, "Identification of the optimal wavelengths for optical topography: A photon measurement density function analysis," J. Biomed. Opt. 15(5), 056002 (2010).

35. S. Powell and T. S. Leung, "Highly parallel Monte-Carlo simulations of the acousto-optic effect in heterogeneous turbid media," J. Biomed. Opt. (under review) (2011).

36. S. Sakadzic and L. V. Wang, "Ultrasonic modulation of multiply scattered coherent light: An analytical model for anisotropically scattering media," Phys. Rev. E. 66(2), 026603 (2002).
37. Y. Li, P. Hemmer, C. Kim, H. Zhang, and L. V. Wang, "Detection of ultrasound-modulated diffuse photons using spectral-hole burning," Opt. Express 16(19), 14862-14874 (2008).

38. C. Kim, R. J. Zemp, and L. V. Wang, "Intense acoustic bursts as a signal-enhancement mechanism in ultrasound-modulated optical tomography," Opt. Lett. 31(16), 2423-2425 (2006).

39. R. Li, D. S. Elson, C. Dunsby, R. Eckersley, and M. X. Tang, "Effects of acoustic radiation force and shear waves for absorption and stiffness sensing in ultrasound modulated optical tomography," Opt. Express 19(8), 7299-7311 (2011).

40. K. Yoshitani, M. Kawaguchi, K. Tatsumi, K. Kitaguchi, and H. Furuya, "A comparison of the INVOS 4100 and the NIRO 300 near-infrared spectrophotometers," Anesth. Analg. 94(3), 586-590 (2002).

41. D. MacLeod, K. Ikeda, J. Keifer, E. Moretti, and W. Ames, "Validation of the CAS adult cerebral oximeter during hypoxia in healthy volunteers," IARS 80th Clinical and Scientific Congress, Anesth. Analg. 102, S162 (2006).

42. M. Calderon-Arnulphi, A. Alaraj, S. Amin-Hanjani, W. W. Mantulin, C. M. Polzonetti, E. Gratton, and F. T. Charbel, "Detection of cerebral ischemia in neurovascular surgery using quantitative frequencydomain near-infrared spectroscopy," J. Neurosurg. 106(2), 283-290 (2007).

43. E. Ohmae, Y. Ouchi, M. Oda, T. Suzuki, S. Nobesawa, T. Kanno, E. Yoshikawa, M. Futatsubashi, Y. Ueda, H. Okada, and Y. Yamashita, "Cerebral hemodynamics evaluation by near-infrared time-resolved spectroscopy: Correlation with simultaneous positron emission tomography measurements," NeuroImage 29(3), 697-705 (2006).

44. A. Liebert, H. Wabnitz, J. Steinbrink, H. Obrig, M. Möller, R. Macdonald, A. Villringer, and H. Rinneberg, "Time-resolved multidistance near-infrared spectroscopy of the adult head: Intracerebral and extracerebral absorption changes from moments of distribution of times of flight of photons," Appl. Opt. 43(15), 3037-3047 (2004).

45. D. T. Delpy, M. Cope, P. Van der Zee, S. Arridge, S. Wray, and J. Wyatt, "Estimation of optical pathlength through tissue from direct time of flight measurement," Phys. Med. Biol. 33(12), 1433-1442 (1988). 